Julia Gruhlich*

\title{
Wer steuert Diversity Management? - Die Akteure im organisationalen Umsetzungsprozess von Gender Diversity am Beispiel eines transnationalen Unternehmens**
}

\section{Zusammenfassung}

Im Gegensatz zu Ansätzen, die Diversity Management als eine Win-Win-Situation für alle betrieblichen Akteure beschreiben, wird mit Bezug auf eine empirische Studie in einem transnationalen Konzern und basierend auf Bourdieus Feld-Habitus-Theorie im Artikel argumentiert, dass die Umsetzung von Diversity Management stark feld- und akteursgebunden ist und damit vor allem der Stärkung von einzelnen Interessen dient. So wird Diversity Management vornehmlich im Management implementiert und richtet sich vorwiegend an weibliche Fach- und Führungskräfte in den Stammländern des transnationalen Unternehmens, während weitere zentrale betriebliche Akteure und Akteursgruppen wie z.B. Gewerkschaften, Betriebsräte und AGG-Beschwerdestellen kaum bis gar nicht in die Aushandlungsprozesse involviert sind. Diese einseitige Ausrichtung und Umsetzung zu überwinden, ist voraussetzungsvoll und stößt auf organisationale wie kollektive und individuelle Hindernisse.

\section{Who Manages Diversity Management? - The Organizational Implementation Process of Gender Diversity in a Transnational Enterprise}

\section{Abstract}

The article takes a critical view of Diversity Management and by doing so, takes distance from approaches that euphemistically call Diversity Management a win-win-situation for all actors at the workplace. Based on Pierre Bourdieu's field-habitus-theory and data from an empirical study which was conducted in a transnational enterprise it becomes apparent that the implementation of diversity management is bound to certain actors and fields and serves individual interest. Diversity Management is implemented first and foremost on the management level and addresses primarily female professional and managerial staff in the ,home countries' of the enterprises. Other key actors such as trade unions, works councils or AGG-complaints committees barely are consulted or engaged. Overcoming this unilateral

* Dr. Julia Gruhlich, Jg. 1982, wissenschaftliche Mitarbeiterin am Institut für Soziologie an der Georg-AugustUniversität Göttingen, Platz der Göttinger Sieben 3, D-37073 Göttingen.

E-Mail: Julia.Gruhlich@uni-goettingen.de

** Artikel eingegangen: 30.4.2016, revidierte Fassung akzeptiert nach doppelt-blindem Begutachtungsverfahren: 10.01.2017. 
orientation and implementation process entails a process that is ridden with prerequisites and encounters organisational, individual and collective obstacles.

Key words: Diversity Management, Organisation, Gender, Transnationalisation, Transnational Companies, Bourdieu

\section{Einleitung}

Die Diskussion um Diversity Management (DiM) boomt - nicht nur in der Privatwirtschaft, sondern auch im öffentlichen Sektor findet das seit den 1990er Jahren aus den USA kommende personalpolitische Instrument in Deutschland zunehmend Verbreitung und wird in den USA und Europa als neue Form der Gleichstellungs- und Antidiskriminierungsstrategie diskutiert (zur Übersicht Krell, 2014). Die Europäische Kommission ist bestrebt, die Umsetzung von DiM durch die Einrichtung der Internetplattform „Charta der Vielfalt" voranzutreiben. Mit dieser soll der EU-weite Austausch zwischen Organisationen gefördert werden, die Vielfalt befürworten und an Maßnahmen interessiert sind, die Vielfalt und Chancengleichheit am Arbeitsplatz unterstützen und gegen Diskriminierung auf Basis von ethnischer Herkunft, sexueller Ausrichtung, Geschlecht, Alter, einer Behinderung oder der Religion vorgehen (vgl. Europäische Kommission, 2016). Seit 2006 haben sich mehr als 2000 Organisationen des privaten wie öffentlichen Sektors in Deutschland zur „Charta der Vielfalt“ bekannt. Selbst formuliertes Ziel der beteiligten Organisationen ist es, als Vorbild zu fungieren und für vorurteilsfreie Arbeitsumfelder bekannt zu werden. Europaweit waren es im Jahr 2014 über 7.000 Organisationen, die sich Diversity gegenüber - zumindest nach außen - aufgeschlossen zeigen und dadurch signalisieren, dass sie gegen Ungleichheiten und Diskriminierungen vorgehen wollen - ohne, dass damit auch die tatsächliche Ausgestaltung und Umsetzung erfasst wäre. Der Trend zu DiM scheint sich fortzusetzen (Köppel, 2010) - zumindest bei international tätigen Großunternehmen mit Stammsitz in den USA und Europa (Vedder, 2009, S. 122).

Wenig ist bislang über den Umsetzungsprozess von DiM in privatwirtschaftlichen Unternehmen in Europa und vor allem in Deutschland bekannt (vgl. Schiederig, 2013; Tatli, Vassilopoulou, Al Ariss, \& Özbilgin 2012; Losert, 2009; Lederle, 2007; Frohnen, 2005). Die Verbreitung von DiM wird in den wenig vorhandenen Studien meist einseitig mit Bezug auf neo-institutionalistische Ansätze analysiert und damit davon ausgegangen, dass Unternehmensleitungen sich an den als vorbildlich, rational und effektiv geltenden Normen, Erwartungen und Leitbildern der institutionellen Umwelt und dem Verhalten anderer Unternehmensleitungen orientieren. Es komme demnach in Organisationen also schlicht zu Nachahmungseffekten, um - zumindest der Fassade nach - gegenüber der Umwelt als effizient, rational etc. zu gelten und sich auf diese Weise, Legitimität zu verschaffen. Ausgelassen wird aus dieser theoretischen Perspektive, dass die Institutionalisierung von Managementkonzepten immer auch das Resultat innerbetrieblicher Aushandlungsprozesse ist (vgl. Hofbauer, Striedinger, Kreissl, \& Sauer 2015; Riegraf, 2013; Süß, 2009). Dies gilt insbesondere für DiM, da es ein in sich unspezifisches Konzept ist, das in der Praxis definiert und konkretisiert werden kann und muss (vgl. Krell, 2014). Entsprechend werden je nach gesellschaftlichem Kontext, der vorhandenen Unternehmenskultur, den Machtkonstellationen sowie den damit verbundenen Ressourcen und Zielgruppen unterschiedliche Ungleichheitsdimensionen und Diskriminierungstatbestände hervorgehoben. $\mathrm{Zu}$ den typischen, weil weit verbreiteten Di- 
versitätsdimensionen gehören Ethnizität (im US-amerikanischen Raum race), Nationalität, Klasse, Geschlecht, Alter, Religion/Weltanschauung, geistige und körperliche Gesundheit und sexuelle Orientierung (ebd., S. 334). Wonach es sich richtet, dass sich bestimmte Diversitätsdimensionen durchsetzen, ist bislang wenig erforscht. Unklar ist auch, wer in den jeweiligen Organisationen die tragenden betrieblichen Akteure in diesem Veränderungsprozess sind.

In der Literatur zu DiM, die sich größtenteils an die betriebliche Praxis richtet (vgl. Hansen, 2014), wird die Verantwortlichkeit für das personalpolitische Konzept DiM im Sinne eines normativen Sollens relativ eindeutig im Bereich des Personalwesens angesiedelt. Als „personalwirtschaftliche und organisationale Orientierung des Managementhandelns“ (Bruchhagen \& Koall, 2004, S. 4; vgl. Stuber, 2004) zählen zu den für die Umsetzung relevanten Akteuren neben dem Personalwesen vor allem das Management, während bereits die Ebene der Beschäftigten, deren unterschiedliche Ressourcen es laut DiM zu nutzen und im Sinne der Unternehmensziele zu steuern gilt, hier weniger Raum einnehmen. Dies bedeutet, dass besonders Führungskräfte, also eine sehr machtvolle betriebliche Akteursgruppe die Ausgestaltung von Diversity und damit auch die Arbeitsbedingungen der Beschäftigten entlang eigener Interessen „,beeinflusst, organisiert und kontrolliert“ (Vedder, 2005, S. 37). Wenn DiM tatsächlich in dieser Weise implementiert würde, wirft dies notwendigerweise die Frage auf, wie die Beschäftigten und ihre betrieblichen und außerbetrieblichen Interessensvertretungen in die Gestaltung der Arbeitsbedingungen einbezogen werden bzw. Einfluss auf die Gestaltung von DiM nehmen. Im anglo-amerikanischen Raum nehmen einige Studien die Rolle der Gewerkschaften bei der Implementierung von DiM in den Blick. Diese Analysen fokussieren vor dem Hintergrund unterschiedlicher politischer und institutioneller Rahmenbedingungen jedoch meist auf die Diskurse zu DiM (Stringfellow, 2012; Green \& Kirton, 2009; Boxenbaum, 2006; Greene, Kirton, \& Wrench 2005; Kamp \& Hagedorn-Rasmussen, 2004; Wrench, 2004). Folgt man einer gängigen organisationstheoretischen Unterscheidung in talk, verstanden als offizielle, zur Schau gestellte Wirklichkeit, und action, verstanden als praktizierte Wirklichkeit (vgl. Brunsson, 2003/1989), lassen sich nur wenige Studien finden, die sich der Ebene der Aktivitäten in transnationalen Unternehmen widmen (eine Ausnahme bilden die Studien von Özbilgin \& Tatli, 2008, 2009).

Ob die konkrete Umsetzung von DiM ein Resultat des innerorganisatorischen Machtgefüges ist, ob durch DiM eine Verschiebung im organisationalen Machtgefüge erreicht wird oder im Gegenteil bisherige Machtkonstellationen gestützt werden (Krell 1996, S. 347), hängt, folgt man mikropolitischen und handlungstheoretischen Ansätzen (Riegraf, 1996), entscheidend von den an der Umsetzung beteiligten Akteuren und Akteursgruppen und den Kommunikations- und Aushandlungsprozessen innerhalb der Organisationen ab. Die Ausgangsfrage des vorliegenden Beitrags lautet daher: Wer ist in die Ausgestaltung von DiM involviert und welche Interessen und Handlungsstrategien verfolgen die an der Umsetzung von DiM beteiligten Akteure und Akteursgruppen? Die Ausgangsthese ist, dass die symbolische Macht von DiM auch innerorganisational als Motor zu Veränderung genutzt werden kann - allerdings nur von jenen sozialen Akteuren und Akteursgruppen, die die betriebswirtschaftliche Auslegung des Konzepts aufgreifen und es aufgrund ihrer bereits einigermaßen etablierten, aber zugleich immer noch randständigen sozialen Positionen für ihre Zwecke definieren und einsetzen können.

In einem ersten Schritt werden die theoretischen Grundlagen und in einem zweiten Schritt die empirischen Eckdaten der Fallstudie präsentiert. Aufbauend auf den Erkenntnissen aus 
der Fallstudie werden in den anschließenden Schritten die tatsächlichen Handlungsspielräume der jeweiligen Akteure (dazu gehören Führungskräfte, Personalwesen, Betriebsrat, AGG-Beauftragte) im untersuchten Unternehmen analysiert. In der abschließenden Zusammenfassung werden die Erkenntnisse auf die emphatisch bis skeptisch geführte Diskussion zu DiM im Kontext industrieller Beziehungen bezogen.

\section{Organisationale Aushandlungs- und Veränderungsprozesse aus Sicht Bourdieus}

Der theoretische Analyserahmen wird mit Bezug auf Pierre Bourdieus Feld-Habitus-Theorie entwickelt (vgl. Bourdieu \& Wacquant 1996, S. 133), mit der - ähnlich wie mit mikropolitischen Ansätzen - davon ausgegangen werden kann, dass die innerbetrieblichen Akteure und Akteursgruppen je nach ihrer sozialen bzw. betrieblichen Position innerhalb des sozialen Feldes unterschiedlich an DiM interessiert sind, unterschiedlich Zugang haben und unterschiedlich an der Ausgestaltung beteiligt sind.

Das soziale Feld, in diesem Fall das innere Sozialgefüge des Unternehmens, ist nach Bourdieu einem Spielfeld vergleichbar, in welchem die Akteure in Konkurrenz zueinanderstehen und ihre Einsätze setzen, um ihre Position innerhalb des Feldes zu verbessern. Die Position, die die Akteure im sozialen Feld einnehmen, hängt ganz entscheidend von ihrer Kapitalausstattung ab: Der Besitz von Ressourcen bzw. Kapitalien bedeutet nicht nur über eine bestimmte Menge an ökonomischen, kulturellen, sozialen und symbolischen Kapital zu verfügen, sondern auch Einfluss auf die Mechanismen nehmen zu können, die darüber entscheiden, welche Kapitalien in dem jeweiligen Feld anerkannt und legitim sind und wie die Verteilung der Kapitalformen zwischen den konkurrierenden Akteuren und Akteursgruppen erfolgt. Zwei Alternativen sind ihnen zur Positionsverbesserung gegeben: Entweder setzen sie die ihnen zur Verfügung stehenden und im Feld relevanten Ressourcen bzw. Kapitalien, wie das kulturelle Kapital (fachliche und außerfachliche Qualifikationen, Wissen und Kompetenz, Zugang zu Informationen, Titel, Auszeichnungen etc.), das soziale Kapital (soziale Kontakte und Gruppenzugehörigkeit) und das symbolische Kapital (Ruf, Autorität und Status) ein (Bourdieu, 1998, S. 151; Hofbauer, 1992, S. 39) oder aber sie arbeiten darauf hin, die ,immanenten Regeln des Spiels ganz oder teilweise zu verändern“, indem sie beispielsweise die Kapitalsorte der Gegner_innen entwerten und die ihnen zur Verfügung stehenden Kapitalsorten aufwerten (Bourdieu \& Wacquant, 1996, S. 129). Bourdieu betrachtet soziale Felder als Netz an Relationen mehr oder weniger machtvoller Akteure und Akteursgruppen. Davon unterscheidet er das sogenannte „Feld der Macht“, das ebenfalls aus Machtbeziehungen besteht und immer dort entsteht, wo über die Machtstrukturen eines Feldes verhandelt wird (vgl. Barlösius, 2006, S. 114ff.). Die Akteure im Feld der Macht verfügen über die nötige Kapitalausstattung, um gestaltenden Einfluss auf die Strukturen eines Feldes (wie die Relevanz der Kapitalien und ihre Verteilung) zu nehmen.

Bourdieus Theorie bietet den großen Vorteil, neben dem unmittelbaren betrieblichen Feld auch den weiteren gesellschaftlichen Kontext (die Ökonomie, die politische Sphäre etc.) und weitere betriebliche Akteure berücksichtigen zu können, die nicht unmittelbar Mitglieder der Organisation sind (Emirbayer \& Johnson, 2008, S. 2f.). Zu diesen Akteuren gehören u.a. Netzwerke und Interessensorganisationen, wie Branchenverbände, Gewerkschaften, Lobbyorganisationen, informelle Netzwerke und Institutionen sowie - wenn auch indirekt wirk- 
sam - der Arbeitsmarkt (vgl. Funder \& Sproll, 2012, S. 58). Innerhalb wie zwischen diesen Akteursgruppen finden soziale Kämpfe um Macht und Deutungshoheit bzw. Legitimation statt (vgl. Hofbauer et al., 2015, S. 140). Mit Bourdieu lässt sich die Frage formulieren, wie die betriebliche Umsetzung von DiM durch die Machtstrukturen der Organisation und die Spielstrategien der feldspezifischen Akteure erklärt werden kann, die an ihrer Einflussnahme auf die Organisation und ihrer Positionsverbesserung interessiert sind.

\section{Betriebsfallstudie zur Handlungspraxis und Implementierung von Diversity Management}

Die empirische Basis der vorliegenden Überlegungen bildet eine Betriebsfallstudie ${ }^{1}$ in der in Deutschland gelegenen Zentrale eines deutschen Familienunternehmens in der Automobilzuliefererbranche (Gruhlich, 2016a). Das analysierte Unternehmen ist typisch für die Branche und die Organisationsform: Zum einen gilt die Automobilindustrie als klassisch männliche Arbeitsdomäne und dies drückt sich im konkreten Fall in Zahlen aus: Weltweit liegt der Anteil weiblicher Beschäftigter an der Gesamtbelegschaft im Unternehmen bei ca. 11,3 Prozent, deutschlandweit lediglich bei ca. 8,9 Prozent. Damit liegt das Unternehmen deutlich unter dem Durchschnittswert von - ebenfalls niedrigen - 14 Prozent weiblicher Beschäftigter im Jahr 2008 in der Automobilbranche (Kopel \& Weber, 2010, S. 3 und www.genderdax.de). Zum anderen hat das Unternehmen die für die Automobilbranche charakteristische Internationalisierung stark vorangetrieben und auch seine Organisationsstrukturen entsprechend transnationalisiert (zum Transnationalisierungprozess der Automobilindustrie Pries, 1999). Im Forschungszeitraum sind fast eine Viertelmillion Mitarbeiter_innen an 170 Standorten in 38 Ländern für das Unternehmen tätig. Als international operierendes Unternehmen agiert es also zugleich global als auch lokal und bewegt sich damit in dem für transnationale Unternehmen typischen Spannungsverhältnis von Globalisierungs- und Lokalisierungsanforderungen.

1 Bestandteil der zwischen September 2010 und Januar 2011 durchgeführten Fallstudie ist erstens eine umfassende Dokumentenanalyse. Dazu gehörten Organisationsdokumente (Organigramm, Arbeitsplatzbeschreibungen/ Stellenbeschreibungen, Dienstvereinbarungen zu Arbeitszeit etc.), erste gleichstellungspolitische Maßnahmen (z.B. Mentoring, Kindertagesstätte, Konzeptionen zu Diversity Management) und Statistiken (z.B. Informationen zur durchschnittlichen Zahl der Beschäftigten, horizontale und vertikale Geschlechterverteilung auf den verschiedenen Ebenen und Bereichen, durchschnittliche Bezahlung). Zweitens wurden 30 leitfadengestützte Informations- und Expert_innengespräche mit Personaler_innen, Führungskräften und Mitarbeiter_innen geführt, um die objektiven Relationen der sozialen Positionen der Akteure im Unternehmen (Konstellationen von Positionen, relevante Ressourcen und ihre Verteilung usw.) zu rekonstruieren. Kriterien für die Auswahl der Gesprächspartner_innen waren dabei in erster Linie ihre Funktionen im Hinblick auf Personalpolitik und Gleichstellungsmaßnahmen (Personalwesen, einzelne Mitarbeiter_innen, die sich gleichstellungspolitisch hervorgetan haben, Frauenbeauftragte im Betriebsrat und AGG-Beauftragte) sowie ihre formal gegebene Entscheidungs- und Gestaltungsmacht (Führungskräfte). Zusätzlich wurden weitere, berufsbiographische Interviews mit Mitarbeiter innen und Führungskräften geführt, um Einblicke in die Karrierepfade im transnationalen Unternehmen zu erhalten (vgl. dazu ausführlich Gruhlich, 2016a \& 2016b). Im Sinne Bourdieus wurden in der Auswertung, die mit der Dokumentarischen Methode (Bohnsack, 2003) erfolgte, die objektiven Relationen der sozialen Positionen und die an sie gebundenen Handlungsspielräume, Macht- und Entscheidungsmittel bzw., wie Bourdieu es nennt, „Trümpfe“ rekonstruiert. Diese setzen den strukturellen Rahmen für die praktischen Handlungsstrategien und alltäglichen Wahrnehmungsmuster der Akteure und beeinflussen damit die Gestaltung von Diversity Management. 


\subsection{Deutungshoheit weiblicher Führungskräfte: DiM als Frauenförderung}

Zum Forschungszeitraum positioniert sich das Unternehmen nach außen nicht zu Diversity, diskutierte eine mögliche Umsetzung des Konzepts jedoch innerbetrieblich. Entsprechend findet sich (noch) keine eigens für den Umsetzungsprozess von DiM geschaffene Diversity-Verantwortliche oder ganze Diversity-Abteilungen, wie es aus anderen Unternehmen bekannt ist (vgl. Özbilgin \& Tatli, S. 2008). Angestoßen wird die Auseinandersetzung mit DiM im Personalwesen und zwar vor allem durch die wenigen weiblichen Führungskräfte, die in dem männlich dominierten Unternehmen nach wie vor eine Minderheit bilden. Zur Erklärung dafür, dass es vor allem die Personalerinnen sind, die sich für DiM stark machen, kann Bourdieus Feld-Habitus-Konzept fruchtbar gemacht werden: Demnach gibt es zwischen dem (androzentrischem) Feld und den (weiblich verorteten) Habitusformen der Akteurinnen einen Bruch. Wenn die alten habituellen Muster der Wahrnehmung nicht (mehr) passen, setzt das Wundern, das Infragestellen ein und eröffnet damit zugleich die Möglichkeiten für ein Anders-Handeln. Die weiblichen Führungskräfte erfahren in dem männlich geprägten Unternehmen in zweierlei Hinsicht einen habituellen Bruch, der dazu führt, dass sie eher geneigt sind, das für die Mehrheit der Beschäftigten „fraglose Gegebene“ (Schütz 1974/1932, S. 99) in Frage zu stellen und DiM als Instrument des betrieblichen Wandels zu nutzen:

Erstens machen sie die Erfahrung des ,Anderssein“ im Unternehmen, da das männlich konnotierte Feld einen männlichen Körper erwartet, der mit ihrer Hexis als Frau, d.h. ihren einverleibten und relativ dauerhaften weiblichen Dispositionen nicht in Einklang zu bringen ist. Die an ein spezifisches körperliches Auftreten gebundene Männlichkeit gilt in dem Automobilzulieferunternehmen als Norm und wird positiv mit Professionalität assoziiert (vgl. auch Wagels, 2013). Anzügliche Witze, sexistische Herabwürdigungen, Bemerkungen zur Kleidung, die als entweder ,zu weiblich“ oder ,zu männlich“ markiert wird, bis hin zur Infragestellung der persönlichen oder fachlichen Eignung tragen dazu bei, das ,weibliche Andere zu konstruieren und sie von der breiten Masse abzuheben. Aus dieser persönlichen Erfahrung als ,Frau' (und nicht nur oder primär als Fachkraft, Managerin) wahrgenommen zu werden und der daraus resultierenden Sichtbarkeit ziehen diese Führungskräfte jedoch auch Stärke. Sie lernen reflexiv mit dem eigenen Geschlecht umzugehen und setzen sich zum Ziel, zu beweisen, dass, so äußert es eine der befragten weiblichen Führungskräfte, „die Vorurteile, die gegenüber einer Frau bestehen, nicht stimmen“. Dabei solidarisieren sie sich, so diese Interviewte, auch mit anderen Frauen: „Natürlich lebe ich ja etwas dadurch vor, dass ich als Frau in der Organisation und natürlich mit den Frauen in der Organisation auch zeige, dass das Blödsinn ist.“ Verbindend wirkt dabei die eigene ,Betroffenheit'. Ihr Engagement geht jedoch über das Selbstverständnis eines Vorbilds hinaus und bezieht sich auch auf gemeinsame Initiativen. $\mathrm{Zu}$ diesen Initiativen gehören die Gründung eines Betriebskindergartens, ein Mentoring-Programm für Frauen und auch die Diskussionen um die Gründung einer Diversity-Arbeitsgruppe.

Zweitens wurden alle weiblichen Führungskräfte der höheren Ebenen extern rekrutiert, was sich unter anderem auch mit den gläsernen Wänden im Unternehmen erklären lässt, die Frauen am innerbetrieblichen Aufstieg - im Sinne einer klassischen Kaminkarriere - hindern. Sozialisiert in einem anderen Unternehmen bringen sie Denk- und Wahrnehmungsmuster mit, die sich nicht mehr passgenau in die strukturellen und kulturellen Bedingungen des neuen Unternehmens fügen. Auch besitzen sie bereits Vorwissen zu DiM aus anderen Firmen oder haben bereits Erfahrungen mit anderen Organisationskulturen und -strukturen 
gesammelt. Eine Führungskraft äußert im Hinblick auf alternative Arbeitszeitmodelle, dass sie das aus einem schwedischen Unternehmen, bei dem sie zuvor gearbeitet hatte, „halt ganz ganz anders" kenne und entsprechend andere Normvorstellungen verinnerlicht hat. So ruft es bei ihr Verwunderung hervor, dass ihre Kollegen sich nicht zugunsten familiärer Pflichten aus der Arbeit zurückziehen (z.B. früher gehen, um Kinder zu betreuen, in Teilzeit gehen etc.). Mit anderen Worten: Gerade aus ihrer betrieblichen Sozialisation in unterschiedlichen Feldern gewinnen sie an Kapital (vor allem in Form von Wissen und Fähigkeiten) und damit an Einflussmöglichkeit. In diesem Sinne erweisen sich die weniger bruchlosen Karrierewege der weiblichen Führungskräfte als Ressource und nicht, wie sonst häufig problematisiert, als ungünstige Abweichung von der Norm der Kaminkarriere, die frei von Unterbrechungen innerhalb einer Abteilung oder eines Unternehmens erfolgt.

Obgleich diese ,Besonderung' viele der weiblichen Beschäftigten erfahren, sind es vor allem die Führungskräfte unter ihnen, die an der Diskussion um DiM beteiligt sind. Die Mehrheit der weiblichen Beschäftigten verfügt kaum, so ließe sich die Situation erklären, über ausreichend Handlungs- und Gestaltungsmacht, um einen gesamtbetrieblichen Veränderungsprozess, wie ihn DiM vorsieht, anzustoßen. Um strukturelle Veränderungen zu erreichen, braucht es neben dem Wissen und Können vor allem soziale Kontakte, Anerkennung und Reputation sowie ausreichend formale Handlungs- und Gestaltungsmacht. Die Mehrheit der weiblichen Beschäftigten verfügt in dieser Hinsicht jedoch über weniger feldrelevante Ressourcen. Die wenigen weiblichen Beschäftigten sind nicht paritätisch im Unternehmen verteilt, sondern finden sich nur in bestimmten Bereichen (wie Personalwesen oder Öffentlichkeitsarbeit) und Funktionen (Assistentinnen, Sachbearbeiterinnen), verfügen damit über weniger feldrelevante Ressourcen wie Entscheidungsgewalt, soziale Netzwerke, Reputation und Anerkennung. Mit steigender Hierarchieebene sinkt der Frauenanteil, so gibt es im Management lediglich einen Frauenanteil von 3,4 Prozent. Das Machtfeld ist also aufs Engste mit Geschlecht verknüpft. Entsprechend richtet sich das strategische Interesse der mit DiM befassten weiblichen Führungskräfte auf die Umdeutung der androzentrischen Strukturen und der damit zusammenhängenden Ressourcen. Das aus ihrer betrieblichen Position im sozialen Feld nachvollziehbare Ziel ist es, die Spielregeln neu zu definieren und im Zuge dessen die ihnen zur Verfügung stehenden Kapitalsorten aufzuwerten.

Das Interesse der weiblichen Führungskräfte im Personalwesen an DiM lässt sich also als Versuch deuten, die ungleich verteilten Ressourcen neu zu verteilen und zugleich eine Auf- bzw. Abwertung der unterschiedlich verteilten Spieleinsätze vorzunehmen: DiM soll dazu dienen, die Position der marginalisierten und abgewerteten weiblichen Beschäftigten im Unternehmen gegenüber der männlichen Beschäftigten zu verbessern. Dies spiegelt sich auch in der Ausdeutung von Diversity wider: Unter Diversity bündeln diese Akteurinnen primär Maßnahmen zur ,Frauen'- und Familienförderung. Ziel der von ihnen anvisierten Diversity Politik ist es, erstens mehr Frauen in Führungspositionen zu bringen und zu unterstützen (u.a. mittels Frauenquote, Mentoringprogramm) und zweitens die Vereinbarkeit von Beruf und Familie für Eltern zu erleichtern (u.a. durch flexible Arbeitszeitmodelle und betriebliche Kinderbetreuungsmöglichkeiten). Die nun unter ,Diversity ‘ zu bündelnden Maßnahmen sind zum Teil von vorneherein auf bestimmte Personengruppen beschränkt: Quotierungen und Mentoringprogramme richten sich auf Frauen in den hochqualifizierten Berufen der höheren Angestellten- und Führungsebenen, werden also nicht den einfachen Angestellten und Werksarbeiter_innen angeboten. Die betrieblichen Kinderbetreuungsmöglichkeiten richten 
sich zwar formal an alle Beschäftigten, die Plätze sind jedoch gering und Vorrang haben Vollzeiterwerbstätige.

Ein weiterer entscheidender Faktor, um Veränderungsmaßnahmen im Unternehmen durchzusetzen, ist, wie eine Führungskraft es ausdrückt, der „persönliche Einsatz“. Der Unternehmensinhaber erklärt, dass er die Auseinandersetzung mit dem Problem der weiblichen Unterrepräsentanz von Frauen zwar gutheiße (er positioniert sich nach außen sichtbar in Zeitungsinterviews offen für mehr Frauen in seinem „modernen Unternehmen“), jedoch keine organisatorischen Veränderungen anstrebe, um sich systematisch mit dem Problem zu befassen. Für die an Gleichstellungs- und Antidiskriminierungsmaßnahmen Interessierten bedeutet dies, dass sie sich ,on the top' zu den eigentlichen Stellenanforderungen für DiM engagieren müssen. So mag es also kaum verwundern, dass die realisierten Maßnahmen durch weibliche Fachkräfte in einflussreichen Positionen (meist Personalerinnen) initiiert und getragen werden, die über die notwendigen Ressourcen verfügen.

Zwei Schwierigkeiten zeichnen sich bei der betrieblichen ,Übersetzung` des gesellschaftlichen Diskurses um Diversity ab:

(1) Es geht nicht um eine umfassende Personalentwicklung und Kommunikation, einen Wandel von Arbeitszeitgestaltung und Unternehmenskultur oder eine Sensibilisierung der (männlichen) Führungskräfte, sondern primär um die Förderung von statushohen ,Frauen als benachteiligte Gruppe. Die Fokussierung auf die Diversity-Dimension ,Geschlecht" und die damit verbundene betriebliche Setzung, dass Gleichstellung und Vereinbarkeit ein frauenspezifisches Problem sind und auf Seiten männlicher Beschäftigter kein Handlungsbedarf bestünde, geht mit den aus der Forschung bekannten Widerständen einher, bei welchen Frauenförderung als Benachteiligung von ,Männern' kritisiert wird und ,Frauen' schlicht defizitär erscheinen (Riegraf, 1996, S. 31). Mit dieser Setzung wird ignoriert, dass weibliche Beschäftigte nicht in erster Linie ,an sich selbst“, sondern dem männlich geprägten Umfeld scheitern (vgl. Hofbauer 2004) und dass die männlichen Beschäftigten nicht wissen, wie sie mit weiblichen Beschäftigten und der Forderung nach Geschlechtergleichheit umgehen sollen (vgl. Höyng, S. 2002). Die hegemoniale Kultur, welche nicht nur die ausgeschlossene Gruppe, sondern potenziell auch immer Einzelpersonen der dominanten Gruppen benachteiligt, wird auf diese Weise nicht problematisiert.

(2) Es bedarf engagierter Personen, die mit ausreichend individueller Verhandlungsmacht und Kapital (insbesondere mit Qualifikationen sowie formalen und informellen Machpositionen) ausgestattet sind, um sowohl Zeit für ein zusätzliches Arbeitspensum zu haben wie auch ausreichend starken Einfluss auf die Entscheidungen des Unternehmensinhabers nehmen zu können. Wer innerbetrieblich über wenig Ressourcen und Verhandlungsmacht verfügt, dem gelingt es jedoch nicht, die eigenen Interessen entsprechend durchzusetzen. Dies führt dazu, dass DiM tendenziell nur die Interessen von weiblichen Beschäftigten in statushöheren Positionen widerspiegelt (vgl. zur Kritik von DiM als „Elitenprojekt“ Schiederig, 2013, S. 345). Die Differenzen zwischen Frauen geraten dabei aus dem Blick und es wird übersehen, dass das Personal nicht nur entlang der sozialen Kategorie Geschlecht, sondern auch entlang anderer Diversity Dimensionen wie Alter, soziale Herkunft, Ethnizität und vor allem auch betriebliche Positionierung (Arbeiter_innen, einfache Angestellte, Führungskräfte) jeweils unterschiedliche Erfahrungen macht und unterschiedliche Bedarfe hat. Es liegt auch an der Art der Implementierung, die ihren Ausgang im Management des Personalwesens nimmt, 
dass der Schulterschluss zur kollektiven Interessensvertretungen nicht gesucht wird. So geht es um individuelle Betroffenheiten auf Managementebene, die durch einzelne machtvolle Akteurinnen bewältigt werden. Zwar ist damit nicht notwendigerweise ausgeschlossen, dass nicht auch Maßnahmen implementiert werden, die allen Beschäftigten zu Gute kommen, doch ist dies nicht explizit in DiM angelegt.

Wie lässt sich gewährleisten, das DiM in seiner ganzen Breite ausdefiniert und gestaltet wird? Ausgehend von der Standortgebundenheit des Wissens und der Interessen lässt sich die These formulieren, dass erst eine Vielzahl an eingebundenen Akteuren und Akteursperspektiven - dies umfasst die Belegschaft ebenso wie das Management - zu einer stärkeren Ausdifferenzierung von DiM führen wird. Dass auch dies noch unzureichend sein kann, wird im Weiteren anhand der Besonderheit der transnationalen Unternehmensstrukturen deutlich, innerhalb welcher Ressourcen nicht nur vertikal, sondern auch horizontal, also innerhalb der Statusgruppen nach Standorten unterschiedlich verteilt sind.

\subsection{Verankerung von Diversity Management im „Feld der Macht"}

Wie deutlich wurde, lässt sich der Wille und die Macht, Gleichstellungs- oder auch Frauenförderpolitik systematisch umzusetzen, im Personalwesen verorten. Betrieblich legitimiert wird die Auseinandersetzung mit DiM mit der genuinen Aufgabe des Personalwesens, den Personaleinsatz - vor allem auf Ebene der höheren Angestellten - sicher zu stellen. Zum Forschungszeitraum bildet sich im Unternehmen eine sogenannte Diversity-Arbeitsgruppe heraus, an der Personalerinnen unterschiedlicher, aber ausnahmslos deutscher, Standorte beteiligt sind. Ziel der Arbeitsgruppe ist es, DiM im Unternehmen zu verankern und zu diesem Zweck gemeinsame Ziele und eine organisatorische bzw. institutionelle Verankerung zu konzeptionalisieren und einen entsprechenden Finanzierungsplan zu erarbeiten, um die Förderung des Unternehmensinhabers zu gewinnen. In den Diskussionen der Arbeitsgruppe zeichnet sich ab, dass die Verantwortung für die Implementierung von DiM im Personalwesen in der Zentrale liegen soll. Dahinter steht die Idee, dass das Thema vom Personalwesen ausgehend, in alle personalbezogenen Entscheidungsprozesse und Verteilungsmaßnahmen eingebunden werden könnte. Der Fokus auf die in Deutschland gelegene Zentrale erscheint vor dem Hintergrund der betrieblichen Feldstrukturen durchaus nachvollziehbar: Die Zentrale ist - trotz aller Dezentralisierung - nach wie vor das entscheidende Machtzentrum des transnationalen Unternehmens, an dem die strategischen Entscheidungen über den Gesamtkonzern getroffen werden. Für die in der Nähe der Zentrale situierten Führungskräfte bedeutet dies, dass sie in einer relativ machtvollen Position sind: In der geographischen Nähe zur Unternehmensleitung und anderen machtvollen Managern sind sie in der Lage, die Ressourcen zu generieren, die für die Initiierung und Gestaltung von Gleichstellungs- und Antidiskriminierungsmaßnahmen notwendig sind. Entsprechend wenig Interesse besteht bislang von Seiten der DiM-Akteure in der Zentrale an einer Ausweitung auf andere Einheiten des Unternehmensnetzwerks. Was sich in dem hier erforschten Unternehmen bereits abzeichnet, ist auch aus anderen Studien zu national aufgestellten Unternehmen bekannt (vgl. Losert, 2009, S. 198): Situiert sich die Verantwortung für DiM nur am Hauptsitz des Unternehmens, dann werden auch die unter Diversity fallenden Maßnahmen nur an diesem Standort durchgeführt und angeboten, während die dezentralen - auch die deutschen - Niederlassungen beispielsweise kein Mentoringangebot oder eine betriebseigene KiTa erhalten. Es macht demnach einen Unterschied, ob die DiM-Akteure im, wie Bourdieu es nennt, „Feld der Macht“, d.h. 
in der Nähe der Konzernspitze platziert sind, oder an den weniger einflussreichen dezentralen Niederlassungen. Diese Zentrierung der Maßnahmen erscheint umso wahrscheinlicher, nimmt man zudem noch eine transnationale Perspektive ein.

Während das Unternehmen auf strategischer Ebene grundsätzlich anstrebt, dass personalpolitische Konzepte innerhalb des transnationalen Unternehmensnetzwerks vom Zentrum in die Peripherie diffundieren, erscheint die damit einhergehende praktische ,Übersetzungsleistung', aus Perspektive der interviewten Experten_innen, grundsätzlich problematisch. Eine Führungskraft aus dem Personalwesen einer US-amerikanischen Niederlassung erklärt im Interview, welche Schwierigkeiten sie bei den in der deutschen Zentrale entwickelten Personalentwicklungsinstrumenten wahrnimmt:

„[...] Hier in Deutschland wird alles auf Deutsch einwickelt und dann übersetzt man ins Englische und dann gibt's einfach Begrifflichkeiten, die machen eigentlich dann wirklich nicht viel Sinn. [...] Das lässt dann natürlich immer Raum für Interpretation. Also Paradebeispiel für mich ist das Wort directiveness. Das gibt‘s im Englischen nicht. [...] Und dann lernt man irgendwelche neuen Vokabeln und nimmt die irgendwie mit, aber das ist nicht per se, dass man damit was anfangen kann."

Es ist zu erwarten, dass diese Übersetzungsproblematik insbesondere auch DiM betrifft (vgl. Özkazanç-Pan \& Calás, 2015). Aus der Literatur zu DiM ist bereits bekannt, dass der englische Begriff „Diversity“ nicht in allen Ländern auf Verständnis stößt und z.B. in Deutschland sogar Ablehnung hervorrufe, weil er zunächst unverständlich sei. Entsprechend würde oft versucht ein deutsches Äquivalent wie z.B. Chancengleichheit oder Vielfalt zu nutzen (Ditzel, 2015, S. 75ff.). Das ,Übersetzen' umfasst dabei jedoch meist auch kulturelle Praktiken, die durch lokale, nationale und internationale Interessen gerahmt werden (vgl. Hürtgen, 2011). Bei den zu erwartenden Kommunikationsbarrieren geht es demnach nicht nur um fehlende oder fehlerhafte Übersetzungen, sondern auch um divergierende kulturelle Perspektiven und um unterschiedliche organisational verankerte Ausdrucks-, Darstellungs- und Handlungsweisen (vgl. Lüsebrink \& Rampeltshammer, 2013; Frisch \& Schmidtgall, 2016). Die oben zitierte Personalerin äußert sich darüber hinaus auch zu den landesspezifischen Rahmenbedingungen und dem institutionellen Kontext wie z.B. der in dem US-amerikanischen Bundesland bestehenden gesetzlichen Verpflichtung zum Abbau rassistischer Personalpraktiken:

„In Deutschland benutzt man das Thema der Frauen und nennt das Diversity, hier [in den USA] ist Diversity ein anderes Thema. Diversity ist auch Frauen, aber im Wesentlichen ist Diversity auf die unterschiedlichen ethnischen Gruppen gerichtet. Es gibt die african-american, asian-american, hispanic und latino american ... immer anderen american hier. Und das ist eigentlich Diversity hier. [...] Aus deutscher Perspektive ist das irgendwie schwer verständlich.“

Es gibt also landesspezifische wie regionale Besonderheiten zu berücksichtigen, nicht nur im Hinblick auf bestehende Gesetzeslagen, sondern auch mit Blick auf den Arbeitskräftemarkt und die Bedeutung institutionalisierter Interessenvertretungen (vgl. dazu auch die Studien von Schiederig, 2013; Özbilgin \& Tatli, 2008). 


\subsection{Symbolische Inkompabilitäten: Desinteresse des AGG-Beauftragten}

Potentiell ließen sich zwei weitere Akteure identifizieren, die bei der Aushandlung und Umsetzung von DiM eine Rolle spielen könnten, dies aber aus verschiedenen Gründen nicht tun - obwohl in der Literatur ein entsprechender Austausch nahegelegt wird (vgl. Krell, Ortlieb \& Sieben, 2008). Dies sind die AGG-Beschwerdestelle des Unternehmens sowie der Betriebsrat.

Die Einführung des Allgemeinen Gleichbehandlungsgesetzes (AGG) im Jahr 2006 gilt als eine der wichtigsten Rahmenbedingungen für Diversity. Das AGG soll den Beschäftigten einen arbeitsrechtlichen Schutz gegen Diskriminierung auf Basis von ,Rasse“ oder wegen der ethnischen Herkunft, des Geschlechts, der Religion oder Weltanschauung, einer Behinderung, des Alters oder der sexuellen Identität sichern. Mit der Einführung des AGG wurde eine mittlere Führungskraft im Unternehmen zusätzlich zu seinen sonstigen Aufgaben vom Unternehmensinhaber zum AGG-Beauftragten ,ernannt ${ }^{\star 2}$, der als Ansprechpartner bei AGG-relevanten Beschwerden dient ${ }^{3}$. Es handelt sich bei ihm um eine männliche Führungskraft auf mittlerer Ebene aus einem der technischen Bereiche, der zusätzlich zu seinen eigentlichen Aufgaben auch als Ansprechperson bei Diskriminierungserfahrungen für die Beschäftigten aller Bereiche und Ebenen fungiert. Nach eigenen Angaben hatte er weder besonderes Interesse noch spezifische Qualifikationen für diese Position, die er seit fast drei Jahren innehat. Er arbeite auf Abruf, stehe also bei Anfragen und nach Terminvereinbarung zum Gespräch bereit, verfolge darüber hinaus aber kein Interesse an dem Thema Antidiskriminierung und Gleichstellung und strebe keine Fortbildungen oder Schulungen in dem Arbeitsbereich an (wie z.B. (Rechts)beratung und Mediation). Er beschreibt sich selbst als ,alten Hasen“ im Unternehmen und hat keine sozialen Beziehungen zu den ,neuen ${ }^{\text {- }}$ - oftmals weiblichen Führungskräften im Personalwesen aufgebaut, die sich für Diversity interessieren und auch zum Betriebsrat hält er keinen engen Kontakt. Tatsächlich ist er der Idee, das Unternehmen könne sich verändern, gegenüber eher skeptisch eingestellt und erinnert sich gerne an die „gute alte Zeit“, in der „die Männer noch unter sich waren“ und „alles noch etwas unbürokratischer“ ablief. Mit Diversität verknüpft er ethnisch, kulturell oder religiös motivierte Konflikte auf Werksebene, die er sich mit der hohen Diversität der Beschäftigten im Werk erklärt und denen er ein „hohes Aggressions- und Konfliktpotenzial“ zuschreibt. Die Umsetzung des AGG präsentiert er als eine reine Pflichterfüllung, als etwas, das in seinen Worten eben ,irgendjemand“ übernimmt, und das ,gemacht werden muss“.

Dass der AGG-Beauftragte seine Amtsausübung als eine Art Pflichtkür beschreibt, lässt sich durch die rhetorische Rahmung des AGG erklären, die sich deutlich von DiM unterscheidet und mit dieser kaum vereinbar ist: Im AGG geht es um rechtskräftige Bestrafung von Diskriminierung, mit dem Ziel Benachteiligungen abzubauen. Bereits früh hat das Gesetz Widerstand auf Seiten der Arbeitgeber- und Wirtschaftsverbände hervorgerufen (vgl. Sieben \& Schimmelpfeng, 2008). Beim DiM hingegen geht es zwar um die proaktive Verhinderung von Diskriminierung mit dem Ziel, den Gewinn des Unternehmens zu steigern, gleichwohl fällt im Kontext der Diversity-Rhetorik kaum der Begriff der Diskriminierung (vgl. Merx \&Vassilopoulou, 2007). So ist auch in den Gesprächen der an Diversity interes-

2 Unternehmen schaffen nur selten und zudem häufig keine unabhängigen Beschwerdestellen (vgl. Rastetter \& Raasch, 2009)

3 Die Schaffung einer oder mehrerer Beschwerdestellen ist im AGG angeraten, jedoch nicht verpflichtend (vgl. AGG, § 12 Abs. 5 Satz 1). 
sierten Akteurinnen im Personalwesen vielmehr positiv die Rede von „Persönlichkeitsentfaltung“, „Toleranz und Offenheit“, „Anerkennung von Vielfalt“, einem „Klima der Wertschätzung“, von „individuellen Fähigkeiten“ und „Potenzialen“, dem „Nutzen“ und „Wohle des Unternehmens". Anders als beim rechtlich bindenden AGG obliegt es den Unternehmen bei DiM zudem selbst, ob sie es einsetzen, welche Ziele sie sich setzen und welche Maßnahmen sie ergreifen. Das AGG als ,von oben' und ,extern' oktroyiertes Instrument, das sich an Maßstäben der Gerechtigkeit (und nicht primär dem ökonomischen Nutzen) misst, erscheint symbolisch nicht kompatibel zur betrieblichen Logik (vgl. Gruhlich \& Riegraf, 2017). Insofern ein stärkeres Engagement dem AGG-Beauftragten innerbetrieblich keinen Gewinn an Anerkennung, einflussreichen sozialen Netzwerken oder ähnlichen feldrelevanten Kapitalien verspricht und er auch persönlich kein Interesse an grundlegenden Veränderungen betrieblicher Strukturen hat, erscheint sein im Interview rhetorisch zur Schau gestelltes, geringes Engagement machtpolitisch nachvollziehbar.

\subsection{Fehlendes Kapital des Betriebsrats: Diversity Management als ,Überforderung'}

Die Analyse zeigt, dass auch der Betriebsrat nicht als Verantwortlicher im strategischen Gestaltungs- und Umsetzungsprozess von DiM wahrgenommen wird bzw. sich selbst nicht als verantwortlich wahrnimmt (vgl. dies bestätigen auch die Befunde zu national aufgestellten Unternehmen von Losert, 2009 und Schiederig, 2013). Rechtlich ist die Einbindung des Gesamtbetriebsrats dann gefordert, wenn mitbestimmungspflichtige Maßnahmen für das Gesamtunternehmen implementiert werden sollen, bei denen eine durch das Betriebsverfassungsgesetz geregelte Mitbestimmungspflicht der betrieblichen Interessenvertretung vorliegt. ${ }^{4}$ Entsprechend sind die örtlichen Betriebsräte dann in die Verhandlungen um einzelne Diversity-Maßnahmen eingebunden, wenn diese von den jeweiligen regionalen bzw. örtlichen Geschäftsleitungen eingeführt werden sollen (vgl. Losert, 2009, S. 199). Auf meine Nachfrage nach der Beteiligung des Betriebsrats ${ }^{5}$ in dem beforschten Unternehmen wurde deutlich, dass dieser sich über die gesetzlichen Regelungen hinaus für die Belange der Beschäftigten in dem von ihnen als „Elfenbeinturm“ bezeichneten Bereich, d.h. der Angestellten auf den höheren Ebenen (aber unterhalb des Managements), nicht zuständig sieht - dies korrespondiert wiederum mit der Wahrnehmung des Personalwesens, ausschließlich für die Angestellten zuständig zu sein. Im Interview zeigt sich, dass die systematische und professionelle Auseinandersetzung mit der Vielfalt der Beschäftigten im Betriebsrat bisher nicht reflektiert wurde - selbst die vermeintlich auf eine längere Tradition zurückblickende Frauenförderung wird erst seit neuestem als Thema im Betriebsrat behandelt.

Der Betriebsrat setzte sich lange Zeit ausschließlich aus Männern zusammen und erst seit kurzem sind auch zwei Frauen unter den 19 Betriebsratsmitgliedern zu finden. Auf ihre Initiative geht die Gründung eines neuen Ausschusses zurück, der sich sogenannter Frauenthemen annimmt (z.B. Arbeitssicherheit bei Schwangerschaft, Rückkehr nach der Elternzeit). Zuvor wurden diese Themen von einem einzigen Betriebsratsmitglied abgedeckt, der zusätzlich auch für die Arbeitssicherheit zuständig war, also die Rolle des „Frauenbeauftrag-

4 Das Mitbestimmungsrecht des Betriebsrats gilt beispielsweise, wenn Betriebskindergärten eingerichtet werden sollen (vgl. $§ 87$ Abs. 1 Nr. 8 BetrVG).

5 Das Interview wurde mit zwei Vertreter_innen des „Ausschusses für Frauenangelegenheit“ geführt, der in dem Jahr erst neu gegründet wurde. 
ten" als zusätzliche Aufgabe übernommen hatte. Eine der neu gewählten Frauenbeauftragten erklärt, dass sie sich noch orientieren müsse und sich über die konkreten Bedarfe der weiblichen Beschäftigten „noch nicht richtig im Klaren“ sei, da diese bisher nie richtig erfasst wurden, sich aber vorstellen könne, zukünftig Ansprechmöglichkeiten für die Beschäftigten auf Werksebene zu schaffen, um ihnen u.a. bei sexueller Belästigung, Mobbing etc. zur Seite zu stehen. Ihr Fokus liegt - ähnlich wie im Personalwesen - auf Frauen, mit dem Unterschied, dass die Frauenbeauftragte dies rhetorisch auch explizit als Frauenpolitik (und nicht als Diversity-Maßnahme) rahmt.

Deutlich wird im Interview, dass die Themen Antidiskriminierung und Gleichstellung innerhalb des Betriebsrats eine Randstellung einnehmen. Ähnlich wie auch im Personalwesen rücken ,Frauenthemen“ im Betriebsrat erst dann auf die politische Agenda, wenn Frauen vorhanden sind, die über entsprechenden Einfluss verfügen und die sich für diese Themen interessieren bzw. denen auch von außen diesbezüglich eine besondere Kompetenz zugeschrieben wird. So erklärt der Ausschuss für Frauenangelegenheiten: „Frauen wenden sich mit Sorgen ja doch eher leichter Mal an andere Frauen“. Förderlich erscheint auch hier die Gründung eines Kollektivs, das sich als Expertengremium der ,Frauen-Themen' annimmt und den Einzelnen mehr Rückhalt gibt. Die Frauenbeauftragten erläutern: „Mögliche Schwierigkeiten können mit mehreren Leuten zusammen diskutiert werden; wir können dann mit den Vorgesetzten darüber reden, um etwas zu verbessern“. Über Austausch mit anderen Personen, die auf mehr Erfahrung im Kontext der Frauenförderung zurückgreifen können, verfügt der Frauenausschuss hingegen nicht. Für entsprechende Fortbildungen bleibt den Mitgliedern des Ausschusses jedoch nach eigenen Angaben wenig Zeit, da bereits ihr Engagement in der betrieblichen Interessensvertretung mit ihren beruflichen Verpflichtungen und ihren Aufgaben in Haushalt und Familie nur schwer zu vereinbaren sei.

Grundsätzlich mangelt es dem Frauenausschuss an den Kapitalien Zeit, sozialer Vernetzung und Wissen. Die geringe Kapitalausstattung begünstigt, so bestätigen auch andere Studien, eine „Überforderung“ der Betriebsräte (vgl. Losert, 2009, S. 195), für die DiM nur eins von vielen Themen auf der Agenda ist. Im Gespräch problematisieren die Interviewten zudem, dass die ausgeprägt männliche Organisationskultur dazu führe, dass „Frauenthemen“ auch innerhalb des Betriebsrats oft randständig bleiben. Entsprechend schwierig bis unmöglich erscheint die bei den Gewerkschaften und in der Literatur zu findende Forderung (ver.di, 2012, S. 104), Betriebsräte sollten die Diversity-Prozesse offensiv nutzen und eine Kontrollfunktion über die Vielzahl an Diversity-Themen ${ }^{6}$ einnehmen, um sicher zu stellen, „,dass ein Diversity Management, einmal vom Vorstand oder der Firmenleitung propagiert, mehr ist, als ein PR-Gag oder eine bloße Überstülpung von Absichtserklärungen" (Haselier \& Thiel, 2005, S. 74).

\section{Fazit}

Wiegt man nun die Potenziale und Gefahren von DiM ab, so zeigt sich zum einen, dass DiM gegenwärtig durch seine hohe symbolische Strahlkraft eine außerordentliche Möglichkeit

6 Wie z.B. Durchsetzung der tatsächlichen Gleichstellung von Frauen und Männern, Vereinbarkeit von Familie und Erwerbstätigkeit, Eingliederung Schwerbehinderter, Beschäftigungsförderung älterer Arbeitnehmer, Integration ausländischer Arbeitnehmer, Bekämpfung von Rassismus und Fremdenfeindlichkeit sowie Überwachung der Einhaltung des AGG (vgl. BetrVG §80). 
bietet, geschlechtliche Ungleichheit in Unternehmen zu thematisieren und das Machtfeld zugunsten von weiblichen Beschäftigten zu verschieben. Am analysierten Fallbeispiel wurde deutlich, dass DiM vor allem von weiblichen Fach- und Führungskräften im Bereich des Personalwesens in der Unternehmenszentrale aufgegriffen wird, die damit maßgeblich an der inhaltlichen Ausformulierung und Gestaltung von Diversity-Maßnahmen beteiligt sind, während einfache Angestellte, Arbeiter_innen, AGG-Beauftragte und Betriebsräte sowie die weltweit verstreuten Konzerneinheiten kaum involviert sind. Erklären lässt sich dies damit, dass sie als Führungskräfte über die entscheidenden Kapitalien verfügen, um DiM zentral zu platzieren. Ihr Interesse an DiM resultiert daraus, dass sie in dem männlich dominierten Feld des Managements in der Automobilbranche die Erfahrung gemacht haben, als ,Frau wahrgenommen zu werden und ungleich behandelt bzw. benachteiligt zu werden. Es liegt im Interesse der weiblichen Führungskräfte die betrieblichen Spielregeln, in denen Männlichkeit als zentrale Karriereressource gilt, zu verändern und Weiblichkeit aufzuwerten. Da Diversity zudem diskursiv bereits als moderne Form der Geschlechterpolitik und zugleich als anerkanntes personalpolitisches Instrument gerahmt ist, erweist es sich gerade für die oftmals bereits zumindest in Teilen etablierten Maßnahmen der Frauenförderung sowie die Interessen des Personalwesens als hoch anschlussfähig.

Die Gefahr, die sich andererseits abzeichnet ist, dass die wenigen betrieblichen Diversity-Reputationsprojekte meist auf die oberen Beschäftigungsebenen gerichtet sind und dabei besonders Frauen in Führungspositionen in einem national begrenzten Kontext adressieren, andere Interessensgruppen vernachlässigen und diese in der Aushandlung mit dem Management und der Konzernzentrale schwächen. Während DiM damit u.U. einen Beitrag zur Verbesserung der Karriere- und Arbeitssituation von ,weißen', hochqualifizierten weiblichen Beschäftigten in höheren Positionen leisten und somit durchaus zum Abbau geschlechtsspezifischer Ungleichheiten im Management beitragen kann, bleiben andere Ungleichheiten im organisationalen Feld unangetastet. Anzunehmen ist, dass ein DiM, das lediglich ein neues Label für eine wiederbelebte Frauen- und Familienförderung ist und das die Vielfalt unterschiedlicher weiblicher wie männlicher Lebensformen nicht adressiert, nicht nur auf Seiten der Männer, die für einen umfassenden organisationalen Wandel ganz entscheidende Akteure darstellen, sondern auch auf Seiten der sozial unterschiedlich positionierten Frauen auf wenig Akzeptanz stößt. Vor dem Hintergrund der Diversität innerhalb wie zwischen den Geschlechtern, weist Jeff Hearn (2014) zu Recht darauf hin, dass der methodologische Nationalismus für transnationale Unternehmen nicht weiterführt, um die Persistenz von Männern im Management zu erklären und - so ließe sich ergänzen - auch nicht um diese Homogenität aufzubrechen. Die selbstkritische Infragestellung, weißer` Hegemonien und Privilegien kann an dieser Stelle neue Perspektiven für DiM wie auch die Organisationsforschung eröffnen (vgl. Al Ariss, Özbilgin, Tatli \& April, 2014).

Versucht man nun ein mögliches Szenario für eine gelungene Umsetzung von DiM in Organisationen abzuleiten, so sähe dies die Beteiligung (z.B. in Form einer Diversity-Arbeits- oder Steuerungsgruppe) vieler Akteure unterschiedlicher Status- und Interessensgruppen, Abteilungen, Niederlassungen, und mit möglichst breiter sozialer Diversität (im Hinblick auf Geschlecht, Alter, Nationalität) vor, die mit ihrem Fachwissen, ihrer jeweiligen Perspektiven und Interessen Probleme explizit machen, Maßnahmen vorschlagen und Ziele entwickeln können. Die Mitwirkung aller organisational relevanten Akteure erfolgt jedoch in ihrer jeweiligen Eigenwilligkeit, d.h. vor dem Hintergrund ihrer jeweiligen sozialen Position im organisationalen Gefüge, ihrer Kapitalausstattung und mit Blick auf die Felder, denen 
sie zugehörig sind und deren Akteuren gegenüber sie ihre Tätigkeit legitimieren müssen. Damit ist die Tätigkeit einer durchmischten Diversity-Arbeits- oder Steuerungsgruppe nicht frei von Hierarchien und bestimmt von ungleicher Ressourcenverteilung, deren Explizitmachung und Ausgleich bzw. Abfederung eine entscheidende Voraussetzung für das Gelingen der Zusammenarbeit darstellt. Das heißt auch, dass die in der betriebswirtschaftlichen Literatur verbreitete Vorstellung eines organisationalen Lern- und Veränderungsprozesses, der in eindeutig definierten Phasen oder vorhersehbaren Schritten (vgl. Keil, 2009) zwischen gleichgestellten Akteuren abläuft, rein idealtypisch ist. Mit dem hier entworfenen Szenario ist also ein - zumindest teilweiser - Abschied von der Machbarkeits- und Steuerungsillusion verbunden: Ebenso wie die Homogenität in Unternehmen auf komplexe Ursachen zurückzuführen ist, lässt sich auch Diversity nicht unilinear von oben in einem zügig durchgeführten Veränderungsprozess steuern, sondern bedarf offener Suchbewegungen der unterschiedlichen Akteure und Akteursgruppen. Die Diversity-Maßnahmen müssen sich im transnationalen Unternehmen zudem letztlich daran messen lassen, inwiefern sie die Organisation als Ganzes sowie die formellen wie informelle Ebenen der Organisation in ihrer Komplexität und Widersprüchlichkeit in den Blick nehmen, was auch impliziert, dass kaum universale Lösungen für alle Standorte möglich sind und Entwicklungen innerhalb der unterschiedlichen Standorte durchaus ambivalent, widersprüchlich und ungleichzeitig erfolgen können.

\section{Literatur}

Al Ariss, A., Özbilgin, M., Tatli, A. \& April, K. (2014). Tackling Whiteness in organizations and management. Journal of managerial psychology 29 (4), 362-369. https://doi.org/10.1108/JMP-102013-0331

Barlösius, E. (2006). Pierre Bourdieu. Frankfurt am Main: Campus.

Bohnsack, R. (2003). Rekonstruktive Sozialforschung. Einführung in qualitative Methoden (5. Aufl.). Opladen: Leske + Budrich. https://doi.org/10.1007/978-3-322-89614-8

Bourdieu, P. (1998). Praktische Vernunft. Zur Theorie des Handelns. Frankfurt am Main: Suhrkamp.

Bourdieu, P. \& Wacquant, L. (1996). Die Ziele der reflexiven Soziologie. In P. Bourdieu \& L. Wacquant, (Hrsg.), Reflexive Anthropologie (95-251). Frankfurt am Main: Suhrkamp.

Boxenbaum, E. (2006). Lost in translation? The making of Danish diversity management. American Behavioral Scientist 49/7, 939-948. Doi 10.1177/0002764205285173

Bruchhagen, V. \& Koall, I. (2004). Managing Diversity, Ein (kritisches) Konzept zur produktiven Nutzung sozialer Differenzen. In R. Becker \& B. Kortendieck (Hrsg.), Handbuch. Frauen- und Geschlechterforschung (S. 931-938). Wiesbaden: VS Verlag.

Brunsson, N. (2003/1989). The Organization of Hypocrisy, Talk, Decisions and Actions in Organization (2. Aufl.). Copenhagen Business School Press.

Ditzel, U. (2015). Das Diversity Management in Deutschland. Hamburg: Igel Verlag.

Emirbayer, M. \& Victoria, J. (2008). Bourdieu and Organizational Analysis. Theory and Society 37/1, 1-44. doi 10.1007/s11186-007-9052-y

Europäische Kommission (2016). Chartas der Vielfalt in der EU. Abgerufen am 14.04.2016. Verfügbar unter http://ec.europa.eu/justice/discrimination/diversity/charters/index de.htm.

Frisch, J. \& Schmidtgall, T. (2016). Interkulturelle Kommunikation in NGOs. Working Papers der Hans-Böckler-Stiftung 004, Düsseldorf.

Frohnen, A. (2005). Diversity in Action. Multinationalität in globalen Unternehmen am Beispiel Ford. Bielefeld: transcript. doi 10.14361/9783839403778

Funder, M. \& Sproll, M. (2012). Symbolische Gewalt und Leistungsregime, Geschlechterungleichheit in der betrieblichen Arbeitspolitik. Münster: Westfälisches Dampfboot. 
Greene, A.M. \& Kirton, G. (2009). Diversity Management in the UK, Organizational and Stakeholder Experiences. London: Routledge.

Greene, A.-M., Kirton, G. \& Wrench, J. (2005). Trade Union Perspectives on Diversity Management, A Comparison of the UK and Denmark. European Journal of Industrial Relations 11(2), 179-196. doi 10.1177/0959680105053962

Gruhlich, J. (2016a). Transnationale Unternehmen und Geschlecht. Eine praxeologische Organisationsanalyse. Reihe Geschlecht und Gesellschaft. Wiesbaden: VS Verlag. https://doi.org/10.1007/9783-658-12336-9

Gruhlich, J. (2016b). Von Car-Guys, Expatriates und Power-Frauen - Empirische Befunde zu Geschlecht und Karrieren im transnationalen Management. In D. Lengersdorf \& M. Meuser (Hrsg.), Männlichkeiten und der Strukturwandel von Erwerbsarbeit in globalisierten Gesellschaften Diagnosen und Perspektiven (S. 55-73). Weinheim/Basel: Beltz Juventa.

Gruhlich, J. \& Riegraf, B. (2017). Gender und Diversity, Theoretische Überlegungen zur Kategorie Geschlecht und Konsequenzen für Gleichstellungspolitiken. In T. Ringeisen \& P. Genkova (Hrsg.), Handbuch Diversity Kompetenz. Wiesbaden: VS Verlag. (im Erscheinen)

Hansen, K. (2014). (Hrsd.). CSR und Diversity Management, Erfolgreiche Vielfalt in Organisationen. Berlin/Heidelberg: Springer Gabler.

Haselier, J. \& Thiel, M. (2005). Diversity Management, Unternehmerische Stärke durch personelle Vielfalt. Handbücher für die Unternehmenspraxis. Frankfurt am Main: Bund-Verlag.

Hearn, J. (2014). Contextualising Men, Masculinities, Leadership and Management, Gender/Intersectionalities, Local/Transnational, Embodied/Virtual, Theory/Practice. In S. Kumra, R. Simpson \& R. J. Burke (Hrsg.), The Oxford Handbook of Gender in Organizations (417-437). Oxford: Oxford University Press.

Hofbauer, J. (1992). Der soziale Raum „Betrieb“. Zur Strukturierung der betrieblichen Sozialwelt aus der Sicht der Bourdieuschen Sozialtheorie. Berlin: Wissenschaftszentrum Berlin für Sozialforschung (WZB).

Hofbauer, J., Striedinger, A., Kreissl, K. \& Sauer, B. (2015). Of Trump Cards and Game Moves, Positioning Gender Equality as an Element of Power Struggles in Universities. In A. Tatli. \& M. Özbilgin (Hrsg.), Pierre Bourdieu, Organization, and Management (S. 139-162). New York: Routledge.

Hürtgen, S. (2011). Europäische Interessenvertretung, eine Frage der nationalen Kultur? Industrielle Beziehungen. Zeitschrift für Arbeit, Organisation und Management 18(4), 315-335. http://nbn-resolving.de/urn:nbn:de:0168-ssoar-342923

Kamp, A. \& Hagedorn-Rasmussen, P. (2004). Diversity management in a Danish context, Towards a multicultural or segregated working life? Economic and Industrial Democracy 25(4), 525-554. doi 10.1177/0143831X04047158

Keil, M. (2009). Diversity Management als Veränderungsprozess. Diversity für mehr Innovation! Migrationspolitisches Portal der Heinrich Böll Stiftung. Abgerufen von https://heimatkunde.boell. de/2009/05/01/diversity-management-als-veraenderungsprozess

Kopel, M. \& Weber, A. (2010). Frauenbeschäftigung in der Automobilindustrie. Entwicklung und aktuelle Situation. Hrsg. v. IG Metall. Abgerufen von http://www.igmetall.de/internet/0157932_tabellenanhang_automobilindustrie_10032010_03e19831e038b675c1d68624a96624249761ea78.pdf

Köppel, P. (2010). Diversity Management in Deutschland, Ein Benchmark unter den DAX 30-Unternehmen. Abgerufen am 09.08.2011. Abgerufen von http: //www.synergyconsult.de/pdf/Benchmark Diversity_Management_DAX30.pdf.

Krell, G. (1996). Mono- oder multikulturelle Organisationen? „Managing Diversity“ auf dem Prüfstand. Industrielle Beziehungen 3(4), 334-350.

Krell, G. (2014). Gender und Diversity, Eine Diskursgeschichte. Funder, M. (Hrsg.), Gender CageRevistited. Handbuch zur Organisations- und Geschlechterforschung (S. 319-343). Baden Baden: Nomos.

Krell, G., Ortlieb, R. \& Sieben, B. (Hrsg.). (2008). Chancengleichheit durch Personalpolitik. Gleichstellung von Männern und Frauen in Unternehmen und Verwaltungen. Rechtliche Regelungen- 
Problemanalysen - Lösungen (5. Aufl.). Wiesbaden: Springer Gabler. doi 10.1007/978-3-83499560-5

Lederle, S. (2007). Die Einführung von Diversity Management in deutschen Organisationen. Eine neoinstitutionalistische Perspektive. Zeitschrift für Personalforschung 21(1), $22-41$.

Losert, A. (2009). Perspektiven auf Diversity Management, Beschäftigte - Betriebsrat - Management. Hamburg: Universität Hamburg.

Lüsebrink, H.-J. \& Rampeltshammer, L. (2013). Interkulturelle Kommunikation in transnationalen Arbeitnehmerinteressenvertretungen - Problemfelder, Institutionelle Rahmenbedingungen, Forschungsperspektiven. In S. Rüb \& T. Müller (Hrsg.), Arbeitsbeziehungen im Prozess der Globalisierung und Europäischen Integration (123-138). Baden Baden: Nomos.

Mense-Petermann, U. \& Wagner, G. (2006). Zur Einleitung, Transnationale Konzerne als neuer Organisationstyp? Glokalität als Organisationsproblem. In U. Mense-Petermann \& G. Wagner (Hrsg.), Transnationale Konzerne als neuer Organisationstyp? Glokalität als Organisationsproblem (S. 9-31). Wiesbaden: VS Verlag.

Merx, A. \& Vassilopoulou, J. (2007). Das arbeitsrechtliche AGG und Diversity-Perspektiven. In I. Koall,, V. Bruchhagen \& F. Höher (Hrsg.), Diversity Outlooks. Diversity zwischen Antidiskriminierung, Ethik und Profit (354-385). Münster: LIT Verlag.

Özbilgin, M. \& Tatli, A. (2008). Global Diversity management. An evidence-based approach. New York: Palgrave Macmillan.

Özbilgin, M. \& Tatli, A. (2009). Understanding Diversity Managers' Role in Organizational Change, Towards a Conceptual Framework. Canadian Journal of Administrative Sciences 26, 244-258. doi 10.1002/cjas. 107

Özkazanç-Pan, B. \& Calás, B. (2015). From Here to There and Back Again. Transnational Perspectives on Diversity in Organizations. In R. Bendl, I. Bleijenbergh, E. Henttonen \& A. J. Mills (Hrsg.), The Oxford Handbook of Diversity in Organizations (S. 575-603). Oxford: University Press.

Pries, L. (1999). Auf dem Weg zu global operierenden Konzernen? BMW, Daimler-Benz und Volkswagen. Die drei Großen der deutschen Automobilindustrie. München und Mering: Rainer Hampp.

Rastetter, D. \& Raasch, S. (2009). Das Allgemeine Gleichbehandlungsgesetz (AGG) als Sparversion - Ergebnisse einer Unternehmensbefragung. Arbeit. Zeitschrift für Arbeitsforschung, Arbeitsgestaltung und Arbeitspolitik 18 (3), 186-199.

Riegraf, B. (1996). Geschlecht und Mikropolitik, Das Beispiel betrieblicher Gleichstellung. Opladen: Leske + Budrich

Riegraf, B. (2008). Managing Diversity - Gleichstellung von Frau und Mann. Endlich voll integriert oder nur noch Nebensache? DjbZ - Zeitschrift des Deutschen Juristinnenverbandes, Schwerpunktheft „, Gender Mainstreaming und Managing Diversity, Alternativen zu Antidiskriminierungsrecht und Gleichstellungsgesetzen im Arbeitsleben?" 11 (1), 23-25.

Riegraf, B. (2013). Frauenförderung als mikropolitische Aushandlungs- und Entscheidungsprozesse in Unternehmen. In U. Müller, B. Riegraf \& S. Wilz (Hrsg.), Geschlecht und Organisation (S. 165183). Wiesbaden: VS Verlag.

Schiederig, K. (2013). Mythos Diversity. Personalpolitik in transnationalen Unternehmen. Frankfurt am Main: Campus-Verlag.

Schütz, A. (1974/1932). Der sinnhafte Aufbau der sozialen Welt. Eine Einleitung in die verstehende Soziologie. Frankfurt am Main: Suhrkamp.

Sieben, B. \& Schimmelpfeng, O. (2008). Forschungsskizze, Gleichstellungspolitik nach dem AGG. Eine Befragung der 100 wertschöpfungsstärksten deutschen Unternehmen. In G. Krell, R. Ortlieb \& B. Sieben (Hrsg.), Chancengleichheit durch Personalpolitik. Gleichstellung von Männern und Frauen in Unternehmen und Verwaltungen. Rechtliche Regelungen - Problemanalysen - Lösungen (5. Aufl., S. 59-65). Wiesbaden: Springer Gabler.

Stringfellow, E. (2012). Trade unions and discourses of diversity management, A comparison of Sweden and Germany. European Journal of Industrial Relations 17, 1-17. doi 10.1177/0959680112461094

Stuber, M. (2004). Diversity, Das Potenzial von Vielfalt nutzen - den Erfolg durch Offenheit steigern. München: Luchterhand. 
Süß, S. (2009). Die Institutionalisierung von Managementkonzepten. Diversity-Management in Deutschland. München und Mering: Hampp Verlag.

Tatli, A., Vassilopoulou, J., Al Ariss, A. \& Özbilgin, M. (2012). The role of regulatory and temporal context in the construction of diversity discourses. The case of the UK, France and Germany. European Journal of Industrial Relations 18 (4), 293-308. doi 10.1515/arbeit-2005-0104

Vedder, G. (2005). Denkanstöße zum Diversity Management. Arbeit-Zeitschrift für Arbeitsforschung, Arbeitsgestaltung und Arbeitspolitik 14 (1), 34-43. doi 10.1515/arbeit-2005-0104

Vedder, G. (2009). Diversity Management, Grundlagen und Entwicklung im internationalen Vergleich. In S. Andresen, M. Koreuber \& D. Lüdke (Hrsg.), Gender und Diversity, Albtraum oder Traumpaar? Interdisziplinärer Dialog zur „Modernisierung“ von Geschlechter- und Gleichstellungspolitik (S. 111-131). Wiesbaden: VS Verlag.

Ver.di (2012 ). Beschlüsse des 3. ver.di-Bundeskongresses vom 17. bis 24. September 2011 in Leipzig. Abgerufen von https: //www.verdi.de/++file++50ac90526f68442e43000000/download/29\%20 05\%20\%20-\%20Beschlussbrosch\%C3\%BCre\%203\%20\%20BuKo.pdf

Wagels, K. (2013). Geschlecht als Artefakt, Regulierungsweisen in Erwerbsarbeitskontexten. Bielefeld: transcript. https://doi.org/10.14361/transcript.9783839422267

Wrench, J. (2004). Trade union responses to immigrants and ethnic inequality in Denmark and the UK, the context of consensus and conflict. European Journal of Industrial Relations 10 (1), 7-30. doi $10.1177 / 0959680104041194$ 


\section{DuEPublico}

Dieser Text wird über DuEPublico, dem Dokumenten- und Publikationsserver der Universität Duisburg-Essen, zur Verfügung gestellt. Die hier veröffentlichte Version der EPublikation kann von einer eventuell ebenfalls veröffentlichten Verlagsversion abweichen.

DOI: $\quad 10.3224 /$ indbez.v24i2.03

URN: urn:nbn:de:hbz:464-20200716-153926-9 